\title{
Joint reconstruction of Stokes images from polarimetric measurements
}

\author{
John R. Valenzuela* and Jeffrey A. Fessler \\ University of Michigan, 1301 Beal Avenue, Ann Arbor, Michigan 48109-2122, USA \\ *Corresponding author: jvalenz@umich.edu
}

Received August 6, 2008; revised November 25, 2008; accepted January 19, 2009; posted February 17, 2009 (Doc. ID 99864); published March 24, 2009

\begin{abstract}
In the field of imaging polarimetry Stokes parameters are sought and must be inferred from noisy and blurred intensity measurements. Using a penalized-likelihood estimation framework we investigate reconstruction quality when estimating intensity images and then transforming to Stokes parameters, and when estimating Stokes parameters directly. We define our cost function for reconstruction by a weighted least-squares data fit term and a regularization penalty. We show that for quadratic regularization the estimators of Stokes and intensity images can be made equal by appropriate choice of regularization parameters. It is empirically shown that, when using edge preserving regularization, estimating the Stokes parameters directly leads to lower RMS error. Also, the addition of a cross channel regularization term further lowers the RMS error for both methods, especially in the case of low SNR. (C) 2009 Optical Society of America

OCIS codes: $100.3020,100.3190,100.3010$.
\end{abstract}

\section{INTRODUCTION}

In remote sensing and astronomical applications the properties of light that are commonly measured and analyzed include intensity, wavelength, and coherence [1,2]. In the context of imaging, intensity measurements provide information on scene content while spectral measurements provide additional information that can be used for material classification and target identification. A fourth property of light that is related to imaging is polarization. Polarization varies slowly with wavelength and so tends to be uncorrelated with spectral measurements [1], thereby offering the potential for image enhancements not available with spectral measurements alone. This paper describes methods for estimating polarization information, i.e., Stokes vectors, from polarimetric intensity measurements.

In the context of remote sensing, polarization signatures are used to infer surface features of an object under incoherent illumination [1,3]. Manmade objects tend to have smoother surfaces than natural objects, so the mechanism of reflection is dominated by specular reflection, which tends to retain or even enhance any polarization of the source.

The state of polarization of a transverse optical field can be represented in several ways [4]; in this paper we focus on the Stokes vector representation. The Stokes vector is a four-component vector $\mathbf{S}=\left(S_{0}, S_{1}, S_{2}, S_{3}\right)$ whose elements are functions of the optical field. The components of the Stokes vector are defined as follows: $S_{0}$ is the total intensity, $S_{1}$ is the difference between the intensity transmitted by a linear polarizer oriented parallel to the $x\left(0^{\circ}\right.$ reference) axis and one oriented parallel to the $y$ axis, $S_{2}$ is the difference between the intensity transmitted by a linear polarizer oriented at $45^{\circ}$ to the $x$ axis and one oriented at $135^{\circ}$, and $S_{3}$ is the difference between the intensity transmitted by a right circular polarizer and a left circular polarizer. In the vast majority of remote sensing applications the component $S_{3}$ is negligible; for this reason it is typical to work with only the first three components of the Stokes vector.

The intensity passed by a linear polarizer whose transmission axis is oriented at angle $\theta$ may be written in terms of the components of the Stokes vector. The effect of an ideal linear polarizer is to pass that part of the electric field that is along the transmission axis of the polarizer. Let the transmission axis of the polarizer be $\mathbf{p}_{\theta}=\cos (\theta) \hat{\mathbf{i}}$ $+\sin (\theta) \hat{\mathbf{j}}$ and the electric field be

$$
\mathbf{E}(t)=E_{x}(t) \hat{\mathbf{i}}+E_{y}(t) \hat{\mathbf{j}} .
$$

The intensity $\Gamma(\theta)$ passed by the polarizer is then

$$
\begin{aligned}
\Gamma(\theta)= & \left\langle\left(\mathbf{E}(t) \cdot \mathbf{p}_{\theta}\right)^{2}\right\rangle \\
= & \left\langle E_{x}^{2}(t)\right\rangle \cos ^{2}(\theta)+\left\langle E_{y}^{2}(t)\right\rangle \sin ^{2}(\theta) \\
& +2\left\langle E_{x}(t) E_{y}(t)\right\rangle \sin (\theta) \cos (\theta),
\end{aligned}
$$

where $\langle\cdot\rangle$ indicates time averaging. Using the double angle formulas we rewrite expression (2) for the intensity as

$$
\begin{aligned}
\Gamma(\theta)= & \frac{1}{2}\left(\left\langle E_{x}^{2}(t)\right\rangle+\left\langle E_{y}^{2}(t)\right\rangle\right)+\frac{1}{2} \cos (2 \theta)\left(\left\langle E_{x}^{2}(t)\right\rangle-\left\langle E_{y}^{2}(t)\right\rangle\right) \\
& +\left\langle E_{x}(t) E_{y}(t)\right\rangle \sin (2 \theta) .
\end{aligned}
$$

The components of the Stokes vector can be written in terms of the electric field as [5]

$$
\begin{aligned}
& S_{0}=\Gamma\left(0^{\circ}\right)+\Gamma\left(90^{\circ}\right)=\left\langle E_{x}^{2}(t)\right\rangle+\left\langle E_{y}^{2}(t)\right\rangle, \\
& S_{1}=\Gamma\left(0^{\circ}\right)-\Gamma\left(90^{\circ}\right)=\left\langle E_{x}^{2}(t)\right\rangle-\left\langle E_{y}^{2}(t)\right\rangle,
\end{aligned}
$$




$$
S_{2}=\Gamma\left(45^{\circ}\right)-\Gamma\left(135^{\circ}\right)=2\left\langle E_{x}(t) E_{y}(t)\right\rangle .
$$

By direct substitution, the intensity is related to the Stokes parameters as

$$
\Gamma(\theta)=\frac{1}{2}\left[S_{0}+S_{1} \cos (2 \theta)+S_{2} \sin (2 \theta)\right] .
$$

For $J$ measurement angles $\left\{\theta_{1}, \ldots, \theta_{J}\right\}$ Eq. (7) becomes a system of $J$ equations. In matrix form the system is

$$
\Gamma=\mathbf{T}_{J \times 3} \mathbf{S} .
$$

where $\boldsymbol{\Gamma}=\left[\left(\Gamma\left(\theta_{1}\right), \ldots, \Gamma\left(\theta_{J}\right)\right]\right.$ and $\mathbf{S}=\left(S_{0}, S_{1}, S_{2}\right)$. The conventional estimate of the Stokes vector uses the pseudoinverse, indicated by ${ }^{\dagger}$, of $\mathbf{T}_{J \times 3}$ :

$$
\hat{\mathbf{S}}=\mathbf{T}_{J \times 3}^{\dagger} \boldsymbol{\Gamma} .
$$

However, the model (7) and the estimate (9) have ignored noise, blur, and other degradations. The proposed methods overcome these limitations.

\section{IMAGE RECONSTRUCTION APPLIED TO STOKES VECTOR IMAGING}

Statistical image reconstruction techniques are applied to data that have been corrupted by nonideal system effects, i.e., noise and blur. When applying a reconstruction algorithm to polarimetric imagery we are confronted with the question of which image set to reconstruct: the polarimetric intensity images or the underlying Stokes images. While access to the Stokes images is the ultimate goal, we must be concerned with the low signal levels in the $S_{1}$ and $S_{2}$ images. On the other hand, the intensity images do not have this low signal difficulty and so are good candidates for improvement through image reconstruction. We investigate both approaches theoretically and numerically. Estimation of the intensity images is referred to as the traditional approach and estimation of the Stokes vector is referred to as the proposed approach. We explore weighted least-squares estimators with both quadratic roughness penalty and edge-preserving regularization.

Our proposed method for Stokes vector estimation can be generalized to account for optical imperfections such as retardances [6], but for notational simplicity we assume the polarization properties of the optical components are ideal here.

\section{A. Traditional Image Restoration Approach}

In the traditional approach to image restoration we try to recover the uncorrupted images from the noisy images individually. For the polarimetric imaging problem this translates into first restoring the true intensity images and then converting those images into Stokes space via the linear transformation (9). We treat the general case of $J$ polarimetric channels (images), each having a unique associated polarization angle.

Denote the lexicographically ordered data collected in the $j$ th channel by $y_{j}$. The system matrix that represents physical effects such as optical and detector blur is denoted $A_{j}$ and the noise vector by $\varepsilon_{j}$. The data vectors $\left\{y_{j}\right\}$ and noise vectors $\varepsilon_{j}$ are each of length $n_{d}$. The size of the system matrix of the $j$ th channel is $n_{d} \times n_{p}$, where $n_{p}$ is the number of pixels in an individual true intensity image $\Gamma_{j}$. (In general, $n_{d} \neq n_{p}$.) The model for the $j$ th channel of the collected data is

$$
y_{j}=A_{j} \Gamma_{j}+\epsilon_{j} .
$$

For simplicity, we adopt a zero-mean iid Gaussian noise model,

$$
\boldsymbol{\epsilon}_{j} \approx N\left(\mathbf{0}, \sigma^{2} \mathbf{I}\right)
$$

Our goal is to estimate the set of intensity images $\boldsymbol{\Gamma}=\left(\Gamma_{1}, \ldots, \Gamma_{J}\right)$ from the set of measurements $\mathbf{y}=\left(y_{1}, \ldots, y_{J}\right)$. Penalized-likelihood, or maximum a posteriori (MAP), estimators have been used extensively for such image reconstruction problems [7-10]. A penalizedlikelihood estimator for $\boldsymbol{\Gamma}$ is given by

$$
\hat{\boldsymbol{\Gamma}}=\underset{\Gamma}{\operatorname{argmin}}\left\{-\log p(\mathbf{y} \mid \boldsymbol{\Gamma})+R_{\Gamma}(\boldsymbol{\Gamma})\right\},
$$

where $R_{\Gamma}$ is a regularization function for the intensity images. Typically, $R_{\Gamma}(\boldsymbol{\Gamma})$ separates, i.e.,

$$
R_{\Gamma}(\boldsymbol{\Gamma})=\sum_{j=1}^{J} R_{j}\left(\Gamma_{j}\right),
$$

and the likelihood also separates, so the minimization problem for $\hat{\boldsymbol{\Gamma}}$ separates into $J$ individual regularized image restoration operations, i.e.,

$$
\hat{\Gamma}_{j}=\underset{\Gamma_{j}}{\operatorname{argmin}}\left\{-\log p\left(y_{j} \mid \Gamma_{j}\right)+R_{j}\left(\Gamma_{j}\right)\right\},
$$

where

$$
-\log p\left(y_{j} \mid \Gamma_{j}\right)=\frac{1}{2 \sigma^{2}}\left\|y_{j}-A_{j} \Gamma_{j}\right\|^{2} .
$$

After restoring each intensity image $\Gamma_{j}$ we then estimate the Stokes vector $\hat{\mathbf{S}}_{\Gamma}$ using a generalized version of Eq. (9) that is appropriate for images. The generalization to images is accomplished by writing the transformation matrix so that we have a pixel-by-pixel version of Eq. (9):

$$
\hat{\mathbf{S}}_{1}=\check{\mathbf{T}}^{\dagger} \hat{\boldsymbol{\Gamma}}=\left(\mathbf{T}_{J \times 3}^{\dagger} \otimes \mathbf{I}_{n_{p}}\right) \hat{\boldsymbol{\Gamma}},
$$

where $\mathbf{I}_{n_{p}}$ is the $n_{p} \times n_{p}$ identity matrix and $\otimes$ denotes Kronecker product.

\section{B. Stokes Estimation Model}

We now propose a method for estimating $\mathbf{S}$ directly from y. The method for estimating the Stokes vector (images) directly differs from from the traditional estimation model in both the likelihood function and the regularization function. is

A penalized-likelihood estimator for the Stokes vector

$$
\hat{\mathbf{S}}=\underset{\mathbf{S}}{\operatorname{argmin}}\left\{-\log p(\mathbf{y} \mid \mathbf{S})+R_{S}(\mathbf{S})\right\}
$$

where $R_{S}$ is a regularization function for the Stokes images. As in the traditional estimator the regularization function $R_{S}$ typically separates, i.e., 


$$
R_{S}(\mathbf{S})=\sum_{j=0}^{2} R_{j}\left(S_{j}\right)
$$

However, unlike the traditional case the likelihood function $p(\mathbf{y} \mid \mathbf{S})$ does not separate, and so the minimization problem of Eq. (17) is coupled. On the other hand, Eq. (17) involves fewer unknown parameters than Eq. (12) because typically $J>3$.

\section{ANALYTICAL ESTIMATOR ANALYSIS: QUADRATICALLY PENALIZED WEIGHTED LEAST-SQUARES ESTIMATOR}

This section compares analytically the traditional and Stokes estimators. The degrading effect of the imaging system is taken to be optical blur and represented by the matrix $B$, i.e., $A_{j}=B$ in Eq. (10). This matrix is Toeplitz if the system is shift invariant, but the analysis that follows applies to a general $B$ matrix. We assume here that each channel has the same optical and detector blur; this is a reasonable assumption, because aberrations and detector effects are not affected by linear polarizers.

We focus on the case of four polarimetric measurements (taken with linear polarizers) at angles $\left[0^{\circ}, 45^{\circ}\right.$, $90^{\circ}, 135^{\circ}$; this sets the size of $\mathbf{T}_{J \times 3}$ in Eq. (8) to be $4 \times 3$, denoted by $\mathbf{T}_{4}$ hereafter. The Stokes-to-intensity transformation $\mathbf{T}_{4}$ and the intensity-to-Stokes transformation $\mathbf{T}_{4}^{\dagger}$ are given by

$$
\mathbf{T}_{4}=\left[\begin{array}{rrr}
\frac{1}{2} & \frac{1}{2} & 0 \\
\frac{1}{2} & 0 & \frac{1}{2} \\
\frac{1}{2} & -\frac{1}{2} & 0 \\
\frac{1}{2} & 0 & -\frac{1}{2}
\end{array}\right], \quad \mathbf{T}_{4}^{\dagger}=\left[\begin{array}{rrrr}
\frac{1}{2} & \frac{1}{2} & \frac{1}{2} & \frac{1}{2} \\
1 & 0 & -1 & 0 \\
0 & 1 & 0 & -1
\end{array}\right] .
$$

We note for use below that $\mathbf{T}_{4}^{\prime} \mathbf{T}_{4}=\operatorname{diag}\left(1, \frac{1}{2}, \frac{1}{2}\right)$, where the prime indicates conjugate transpose. In fact the analysis that follows applies to any set of polarization angles for which $\mathbf{T}_{J \times 3}^{\prime} \mathbf{T}_{J \times 3}$ is a diagonal matrix.

\section{A. Traditional Estimator}

The data model of Eq. (10) becomes

$$
\mathbf{y}=\left(\mathbf{I}_{4} \otimes B\right) \boldsymbol{\Gamma}+\epsilon .
$$

For simplicity we consider a quadratic regularizing penalty function that uses vertical and horizontal neighboring pixels. In one dimension the regularizer $R_{\Gamma}(\Gamma)$ is written

$$
R_{\Gamma}(\Gamma)=\frac{1}{2} \beta \sum_{j=1}^{J} \sum_{k=2}^{n_{p}}\left(\Gamma_{j k}-\Gamma_{j_{k-1}}\right)^{2}=\frac{1}{2} \beta \sum_{j=1}^{J}\left\|C \Gamma_{j}\right\|^{2},
$$

where $C$ is a finite differencing matrix and $\beta$ is the regularization "tuning" parameter. Note that it is reasonable to use the same regularization parameter for the different polarimetric channels because there is a high correlation between them. In two dimensions the summation notation becomes cumbersome and so we use linear algebra notation exclusively. Let $C$ be a finite differencing matrix that takes both vertical and horizontal differences; then the regularization function can be succinctly written, noting that $\mathbf{C} \triangleq \mathbf{I}_{4} \otimes C$, as

$$
R_{\Gamma}(\boldsymbol{\Gamma})=\frac{1}{2} \beta\|\mathbf{C} \boldsymbol{\Gamma}\|^{2}
$$

The estimator (12) can then be written

$$
\hat{\boldsymbol{\Gamma}}=\underset{\Gamma}{\operatorname{argmin}}\left\{\frac{1}{2 \sigma^{2}}\left\|\mathbf{y}-\left(\mathbf{I}_{4} \otimes B\right) \boldsymbol{\Gamma}\right\|^{2}+\frac{1}{2} \beta\|\mathbf{C} \boldsymbol{\Gamma}\|^{2}\right\} .
$$

We consider the case of unconstrained optimization and so the minimizer must satisfy

$$
\nabla_{\Gamma}\left[\frac{1}{2 \sigma^{2}}\left\|\mathbf{y}-\left(\mathbf{I}_{4} \otimes B\right) \boldsymbol{\Gamma}\right\|^{2}+\frac{1}{2} \beta\|\mathbf{C} \boldsymbol{\Gamma}\|^{2}\right]=\mathbf{0} .
$$

Solving the above equation for $\hat{\boldsymbol{\Gamma}}$ and combining with Eq. (16) yields

$$
\hat{\mathbf{S}}_{\Gamma}=\check{\mathbf{T}}^{\dagger}\left\{\mathbf{I}_{4} \otimes\left[\left(B^{\prime} B+\sigma^{2} \beta R\right)^{-1} B^{\prime}\right]\right\} \mathbf{y},
$$

where $R=C^{\prime} C$. This expression corresponds to separate deblurring of each polarimetric channel followed by converting the restored images into Stokes images.

\section{B. Stokes Estimator}

Noting that the system effects are identical to the case of the traditional estimator we write the data model for the Stokes estimator as

$$
\mathbf{y}=\left(\mathbf{T}_{4} \otimes B\right) \mathbf{S}+\epsilon .
$$

Following the same procedure as in the case of the traditional estimator we have the implicitly defined estimator

$$
\begin{aligned}
\hat{\mathbf{S}}= & \underset{\mathbf{S}}{\operatorname{argmin}}\left\{\frac{1}{2 \sigma^{2}}\left\|\mathbf{y}-\left(\mathbf{T}_{4} \otimes B\right) \mathbf{S}\right\|^{2}\right. \\
& \left.+\frac{1}{2}\left(\beta_{0}\left\|C S_{0}\right\|^{2}+\beta_{1}\left\|C S_{1}\right\|^{2}+\beta_{2}\left\|C S_{2}\right\|^{2}\right)\right\} .
\end{aligned}
$$

In this case the three images being estimated are very different and so justify three independent regularization "tuning" parameters. Minimizing with respect to $\mathbf{S}$ leads to the closed form estimator

$$
\hat{\mathbf{S}}=\left(\mathbf{T}_{4}^{\prime} \mathbf{T}_{4} \otimes B^{\prime} B+\sigma^{2} \beta_{3} \otimes R\right)^{-1}\left(\mathbf{T}_{4}^{\prime} \otimes B^{\prime}\right) \mathbf{y}
$$

where $\beta_{3} \triangleq \operatorname{diag}\left(\beta_{0}, \beta_{1}, \beta_{2}\right)$. We now analyze the two approaches (23) and (26).

\section{Spatial Resolution Analysis of the Stokes Estimator} We begin by calculating the mean value of the proposed estimator (26). To aid in the calculation, define $P$ $=\operatorname{diag}(1,2,2)$ and note that $\mathbf{T}_{4}^{\prime} \mathbf{T}_{4}=P^{-1}=\operatorname{diag}\left(1, \frac{1}{2}, \frac{1}{2}\right)$. Then using Eq. (19), 


$$
\begin{aligned}
E[\hat{\mathbf{S}} \mid \mathbf{S}]= & \left(\mathbf{T}_{4}^{\prime} \mathbf{T}_{4} \otimes B^{\prime} B+\sigma^{2} \beta_{3} \otimes R\right)^{-1} \\
& \times\left(\mathbf{I}_{3} \otimes B^{\prime}\right)\left(P^{-1} \otimes \mathbf{I}_{n_{p}}\right)\left(\mathbf{T}_{4}^{\dagger} \otimes \mathbf{I}_{n_{p}}\right) E[\mathbf{y} \mid \mathbf{S}] \\
= & \left(\mathbf{T}_{4}^{\prime} \mathbf{T}_{4} \otimes B^{\prime} B+\sigma^{2} \beta_{3} \otimes R\right)^{-1} \\
& \times\left(\mathbf{I}_{3} \otimes B^{\prime}\right)\left(P^{-1} \otimes \mathbf{I}_{n_{p}}\right)\left(\mathbf{I}_{3} \otimes B\right) \mathbf{S} \\
= & \underbrace{\left(P^{-1} \otimes B^{\prime} B+\sigma^{2} \beta_{3} \otimes R\right)^{-1}\left(P^{-1} \otimes B^{\prime} B\right)}_{\mathbf{L}} \mathbf{S}=\mathbf{L S},
\end{aligned}
$$

where $\mathbf{L}$ is a $\left[3 n_{p} \times 3 n_{p}\right]$ matrix that acts somewhat like a Wiener filter. Each term in the above expression is uncoupled since the matrices $P^{-1}$ and $\beta_{3}$ are diagonal. To explore the spatial resolution properties of the estimators, we approximate $B$ by a circulant matrix. Let $Q$ be the orthonormal discrete Fourier transform (DFT) matrix; then the eigendecompostions $B$ and $C$ are approximated by $Q \Theta Q^{\prime}$ and $Q \Omega Q^{\prime}$, respectively. Then

$$
\begin{aligned}
E[\hat{\mathbf{S}} \mid \mathbf{S}]= & \left(P^{-1} \otimes Q \Theta^{\prime} \Theta Q^{\prime}+\sigma^{2} \beta_{3} \otimes Q \Omega^{\prime} \Omega Q^{\prime}\right)^{-1} \\
& \cdot\left(P^{-1} \otimes Q \Theta^{\prime} \Theta Q^{\prime}\right) \mathbf{S} \\
= & Q\left[\left(P^{-1} \otimes \Theta^{\prime} \Theta+\sigma^{2} \beta_{3} \otimes \Omega^{\prime} \Omega\right)^{-1} \cdot\left(P^{-1} \otimes \Theta^{\prime} \Theta\right)\right] Q^{\prime} \mathbf{S} .
\end{aligned}
$$

We see that the expectation of $\hat{\mathbf{S}}$ is approximately a filtered version of the true objects with each image having an independent filter:

$$
\begin{aligned}
& \text { Filter for } S_{0} \Rightarrow L_{k}=\frac{\left|B_{k}\right|^{2}}{\left|B_{k}\right|^{2}+\beta_{0} \sigma^{2}\left|F_{k}\right|^{2}}, \\
& \text { Filter for } S_{1} \Rightarrow L_{k}=\frac{\frac{1}{2}\left|B_{k}\right|^{2}}{\frac{1}{2}\left|B_{k}\right|^{2}+\beta_{1} \sigma^{2}\left|F_{k}\right|^{2}}, \\
& \text { Filter for } S_{2} \Rightarrow L_{k}=\frac{\frac{1}{2}\left|B_{k}\right|^{2}}{\frac{1}{2}\left|B_{k}\right|^{2}+\beta_{2} \sigma^{2}\left|F_{k}\right|^{2}},
\end{aligned}
$$

where $\left\{B_{k}\right\}$ and $\left\{F_{k}\right\}$ are the DFT coefficients of the first column of $B$ and $C$, respectively, and $k=1, \ldots, n_{p}$. We see that the $S_{1}$ and $S_{2}$ channels have a different spatial resolution than the $S_{0}$ channel unless we choose $\beta_{1}=\beta_{2}$ $=\beta_{0} / 2$. However, in the intensity model all three channels always have identical resolutions.

Matching the spatial resolution of the $S_{1}$ and $S_{2}$ channels to the $S_{0}$ channel also decouples the estimator. That is, by choosing

$$
\beta_{3}=\beta \operatorname{diag}\left(1, \frac{1}{2}, \frac{1}{2}\right)
$$

we have

$$
\begin{aligned}
\hat{\mathbf{S}} & =\left(\mathbf{T}_{4}^{\prime} \mathbf{T}_{4} \otimes B^{\prime} B+\sigma^{2} \beta_{3} \otimes R\right)^{-1}\left(\mathbf{T}_{4}^{\prime} \otimes B^{\prime}\right) \mathbf{y} \\
& =\left[\operatorname{diag}\left(1, \frac{1}{2}, \frac{1}{2}\right) \otimes\left(B^{\prime} B+\sigma^{2} \beta R\right)\right]^{-1}\left(\mathbf{T}_{4}^{\prime} \otimes B^{\prime}\right) \mathbf{y} \\
& =\operatorname{diag}(1,2,2) \otimes\left(B^{\prime} B+\sigma^{2} \beta R\right)^{-1}\left(\mathbf{T}_{4}^{\prime} \otimes B^{\prime}\right) \mathbf{y} \\
& =\mathbf{T}_{4}^{\dagger} \otimes\left[B^{\prime} B+\sigma^{2} \beta R\right]^{-1} B^{\prime} \mathbf{y}=\hat{\mathbf{S}}_{\Gamma} .
\end{aligned}
$$

In other words, for the choice (29), with quadratic regu- larization the Stokes estimator becomes uncoupled and reduces to the traditional estimator.

Next we consider the covariance of the estimator:

$$
\begin{aligned}
\operatorname{Cov}(\hat{\mathbf{S}} \mid \mathbf{S})= & \operatorname{Cov}(\mathbf{L y} \mid \mathbf{S}) \\
= & \sigma^{2}\left(P^{-1} \otimes B^{\prime} B+\sigma^{2} \beta_{3} \otimes R\right)^{-1} \\
& \cdot\left(P^{-1} \otimes B^{\prime} B\right)\left(P^{-1} \otimes B^{\prime} B+\sigma^{2} \beta_{3} \otimes R\right)^{-1} .
\end{aligned}
$$

In the circulant approximation we have

$$
\begin{aligned}
\operatorname{Cov}(\hat{\mathbf{S}} \mid \mathbf{S})= & \sigma^{2}\left(P^{-1} \otimes Q \Theta^{\prime} \Theta Q^{\prime}+\sigma^{2} \beta_{3} \otimes Q \Omega^{\prime} \Omega Q^{\prime}\right)^{-1} \\
& \cdot\left(P^{-1} \otimes Q \Theta^{\prime} \Theta Q^{\prime}\right) \\
& \cdot\left(P^{-1} \otimes Q \Theta^{\prime} \Theta Q^{\prime}+\sigma^{2} \beta_{3} \otimes Q \Omega^{\prime} \Omega Q^{\prime}\right)^{-1} \\
= & \sigma^{2} Q\left(P^{-1} \otimes \Theta^{\prime} \Theta+\sigma^{2} \beta_{3} \Omega^{\prime} \Omega\right)^{-1} \\
& \cdot\left(P^{-1} \otimes \Theta^{\prime} \Theta\right) \cdot\left(P^{-1} \otimes \Theta^{\prime} \Theta+\sigma^{2} \beta_{3} \Omega^{\prime} \Omega\right)^{-1} Q^{\prime} .
\end{aligned}
$$

The variance of the $i$ th pixel in each image is

$$
\begin{aligned}
& \operatorname{Var}\left\{S_{0_{i}} \mid \mathbf{S}\right\}=\frac{\sigma^{2}}{n_{p}} \sum_{k} \frac{\left|B_{k}\right|^{2}}{\left(\left|B_{k}\right|^{2}+\beta_{0} \sigma^{2}\left|F_{k}\right|^{2}\right)^{2}} \\
& \operatorname{Var}\left\{S_{1_{i}} \mid \mathbf{S}\right\}=\frac{\sigma^{2}}{n_{p}} \sum_{k} \frac{2\left|B_{k}\right|^{2}}{\left(\left|B_{k}\right|^{2}+\beta_{1} \sigma^{2}\left|F_{k}\right|^{2}\right)^{2}} \\
& \operatorname{Var}\left\{S_{2_{i}} \mid \mathbf{S}\right\}=\frac{\sigma^{2}}{n_{p}} \sum_{k} \frac{2\left|B_{k}\right|^{2}}{\left(\left|B_{k}\right|^{2}+\beta_{2} \sigma^{2}\left|F_{k}\right|^{2}\right)^{2}}
\end{aligned}
$$

For the choice (29) the noise in the reconstructed Stokes images $\left\{S_{1}, S_{2}\right\}$ is twice that of $S_{0}$, indicating that the regularization for the $\left\{S_{1}, S_{2}\right\}$ images may need to be stronger than $S_{0}$. In summary, if one used quadratic regularization with regularization parameters as in Eq. (29), then $\hat{\mathbf{S}}$ and $\hat{\mathbf{S}}_{\Gamma}$ would be identical. Next we turn to the case of nonquadratic regularization.

\section{EMPIRICAL STUDIES}

\section{A. Edge-Preserving Regularization}

In the Stokes images $\left\{S_{1}, S_{2}\right\}$ the polarization information typically has sharp edges. To recover as much polarization information as possible the regularization function should preserve edges. Since quadratic regularization tends to wash out edges and smooth noise we explore edge-preserving regularization using the hyperbolic function $\psi(t ; \delta)=\delta^{2}\left[\sqrt{1+(t / \delta)^{2}}-1\right]$. For fixed $\delta$ this function is approximately quadratic for values of $t<\delta$ and approximately linear for $t>\delta$. This behavior will tend to smooth noise and preserve edges. The Stokes and intensity regularizers are written

$$
\begin{aligned}
& R_{\Gamma}(\boldsymbol{\Gamma})=\sum_{j} \sum_{k} \psi\left(\left[C \Gamma_{j}\right] ; \delta\right), \\
& R_{S}(\mathbf{S})=\sum_{\ell=0}^{2} \sum_{k} \psi\left(\left[C S_{\ell}\right] ; \delta_{\ell}\right) .
\end{aligned}
$$


The Stokes estimator now has two regularization parameters per Stokes image and the intensity estimator has two regularization parameters in total. Since closed form expressions for the minimizers of these cost functions are intractable we minimize them numerically. The numerical optimization was done with the limited Broyden-Fletcher-Goldfarb-Shanno algorithm [11]. To obtain optimal values of all regularization parameter combinations would be computationally burdensome so we chose the $\beta$ parameters by analyzing the local pointspread function (PSF) [12] of the quadratic estimators. The $\beta$ parameters are chosen so that the FWHM of the estimator is a prescribed amount in regions where the regularization function is approximately quadratic. The local impulse response is defined by

$$
\begin{aligned}
\mathbf{l}_{j}(\mathbf{S}) & =\lim _{\epsilon \rightarrow 0} \frac{\hat{\mathbf{S}}\left[\bar{y}\left(\mathbf{S}+\epsilon \mathbf{e}_{j}\right)\right]-\hat{\mathbf{S}}[\bar{y}(\mathbf{S})]}{\epsilon} \\
& =\nabla \hat{\mathbf{S}}[\bar{y}(\mathbf{S})] \nabla \bar{y}(\mathbf{S}) \mathbf{e}_{j} .
\end{aligned}
$$

For the quadratically penalized, weighted, leastsquares estimator with white Gaussian noise the local impulse response of each Stokes image is independent and written [13]

$$
\mathbf{l}_{k_{j}}=\left(B^{\prime} B+\sigma^{2} \beta_{k} R\right)^{-1} B^{\prime} B \mathbf{e}_{k_{j}},
$$

where $k \in\{0,1,2\}$ indicates the Stokes image. Using a local Fourier approximation we can compute this impulse response with fast Fourier transforms (FFTs). Since the FFTs are computationally inexpensive we can sweep over the $\beta$ parameters and choose the one that corresponds to an $a$ priori FWHM of the impulse response. By choosing $\beta$ so that the estimator has controlled noise smoothing properties we can vary the $\delta$ parameters to find optimal values.

\section{B. Cross-Channel Regularization}

Polarimetric signatures usually are correlated in the Stokes parameters $S_{1}$ and $S_{2}$. To exploit this correlation we can introduce a cross-channel regularization term into the cost function. Cross-channel regularization has proved beneficial in multispectral image restoration algorithms $[8,14]$. The cross-channel regularization functions we adopt for the traditional and proposed estimators are

$$
\begin{aligned}
& R_{\text {cross }}(\boldsymbol{\Gamma})=\beta_{\text {cross }} \sum_{k=1}^{n_{p}} \sqrt{\sum_{j=1}^{J}\left[C \Gamma_{j}\right]_{k}^{2}}, \\
& R_{\text {cross }}(\mathbf{S})=\beta_{\text {cross }} \sum_{k=1}^{n_{p}} \sqrt{\left[C S_{0}\right]_{k}^{2}+\left[C S_{1}\right]_{k}^{2}+\left[C S_{2}\right]_{k}^{2}} .
\end{aligned}
$$

The traditional and Stokes estimators, using channel-bychannel and cross-channel regularization, are then

$$
\begin{aligned}
\hat{\boldsymbol{\Gamma}}=\underset{\boldsymbol{\Gamma}}{\operatorname{argmin}}\left\{\frac{1}{2 \sigma^{2}}\left\|\mathbf{y}-\left(\mathbf{I}_{4} \otimes B\right) \Gamma\right\|^{2}+\beta \sum_{j} \psi\left(C \Gamma_{j} ; \delta\right)\right\} \\
+\beta_{\operatorname{cross}} \sum_{k=1}^{n_{p}} \sqrt{\sum_{j=1}^{4}\left[C \Gamma_{j}\right]_{k}^{2}}, \\
\hat{\mathbf{S}}=\underset{\mathbf{S}}{\operatorname{argmin}}\left\{\frac{1}{2 \sigma^{2}}\left\|\mathbf{y}-\left(\mathbf{T}_{4 \times 3} \otimes B\right) \mathbf{S}\right\|^{2}+\beta_{0} \psi\left(C S_{0} ; \delta_{0}\right)\right. \\
+\beta_{1} \psi\left(C S_{1} ; \delta_{1}\right)+\beta_{2} \psi\left(C S_{2} ; \delta_{2}\right) \\
\left.+\beta_{\operatorname{cross}} \sum_{k=1}^{n_{p}} \sqrt{\left[C S_{0}\right]_{k}^{2}+\left[C S_{1}\right]_{k}^{2}+\left[C S_{2}\right]_{k}^{2}}\right\} .
\end{aligned}
$$

The additional parameters $\beta_{\text {cross }}$ were varied over a range of values to find optimal settings.

\section{SIMULATION EXPERIMENTS}

Simulation experiments were performed to evaluate both the traditional and proposed estimators as well as the cross-channel regularization. For the true imagery we used polarimetric images that were collected by General Dynamics Advanced Information Systems in Ypsilanti,

Table 1. Simulation Results $(\mathrm{SNR}=25 \mathrm{~dB})$ : RMS Error Percentages

\begin{tabular}{ccccc}
\hline & Stokes & Traditional & $\begin{array}{c}\text { Stokes with } \\
\text { Cross-Channel }\end{array}$ & $\begin{array}{c}\text { Traditional with } \\
\text { Cross-Channel }\end{array}$ \\
\hline$\hat{S_{0}}$ & $0.71 \pm 0.0035 \%$ & $0.91 \pm 0.0040 \%$ & $1.07 \pm 0.0035 \%$ & $1.30 \pm 0.0040 \%$ \\
$\hat{S_{1}}$ & $42.19 \pm 0.17 \%$ & $62.45 \pm 0.24 \%$ & $36.97 \pm 0.16 \%$ & $38.57 \pm 0.17 \%$ \\
$\hat{S_{2}}$ & $45.93 \pm 0.19 \%$ & $67.08 \pm 0.25 \%$ & $40.58 \pm 0.17 \%$ & $42.00 \pm 0.19 \%$ \\
DOLP & $27.83 \pm 0.11 \%$ & $39.90 \pm 0.15 \%$ & $24.78 \pm 0.10 \%$ & $24.54 \pm 0.11 \%$ \\
\hline
\end{tabular}

Table 2. Simulation Results ( $\mathrm{SNR}=15 \mathrm{~dB})$ : RMS Error Percentages

\begin{tabular}{ccccc}
\hline & Stokes & Traditional & $\begin{array}{c}\text { Stokes with } \\
\text { Cross-Channel }\end{array}$ & $\begin{array}{c}\text { Traditional with } \\
\text { Cross-Channel }\end{array}$ \\
\hline$\hat{S_{0}}$ & $2.49 \pm 0.011 \%$ & $3.10 \pm 0.015 \%$ & $2.74 \pm 0.012 \%$ & $3.44 \pm 0.015 \%$ \\
$\hat{S_{1}}$ & $61.90 \pm 0.27 \%$ & $126.88 \pm 0.57 \%$ & $58.13 \pm 0.25 \%$ & $69.18 \pm 0.30 \%$ \\
$\hat{S_{2}}$ & $65.35 \pm 0.29 \%$ & $137.32 \pm 0.60 \%$ & $61.14 \pm 0.26 \%$ & $75.00 \pm 0.33 \%$ \\
DOLP & $43.95 \pm 0.20 \%$ & $96.59 \pm 0.31 \%$ & $42.37 \pm 0.18 \%$ & $46.68 \pm 0.20 \%$ \\
\hline
\end{tabular}




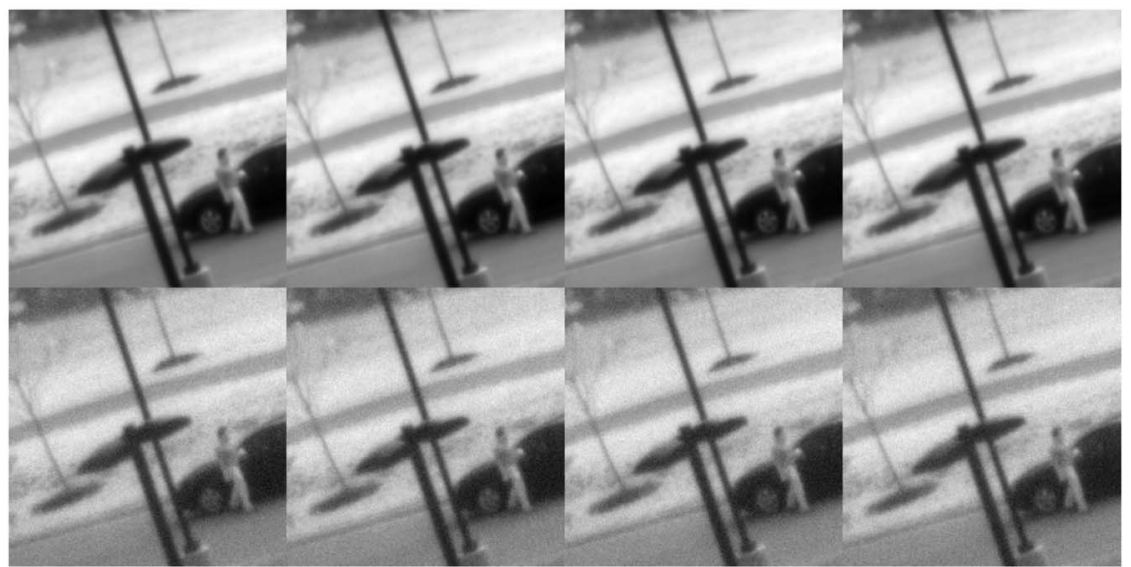

Fig. 1. Noisy and blurred polarimetric imagery. The first row has an SNR of $25 \mathrm{~dB}$ and the second row has an SNR of $15 \mathrm{~dB}$. From left to right the angle of the polarizer is $\left\{0^{\circ}, 45^{\circ}, 90^{\circ}, 135^{\circ}\right\}$.

Michigan; the polarization angles of the sensor were $\left\{0^{\circ}\right.$, $\left.45^{\circ}, 90^{\circ}, 135^{\circ}\right\}$. We added known optical blur and Gaussian noise to the imagery. The system PSF had a FWHM of 1.9 pixels; the PSF was constructed from a phase screen, parameterized by a uniform distribution of the first 20 Zernike polynomials, and placed in an annular pupil. We define the SNR of an image by $\mathrm{SNR}=20 \log _{10}(\|\bar{y}\| / \| \bar{y}$ $-y \|) \mathrm{dB}$, where $\bar{y}$ and $y$ are the uncorrupted and corrupted images, respectively. The simulation experiments were done using the same PSF at two SNR levels, $25 \mathrm{~dB}$ and $15 \mathrm{~dB}$.

The regularization parameters $\left[\beta_{0}, \beta_{1}, \beta_{2}, \beta\right]$ were chosen so that the FWHM of the estimator's PSF was 1.5 pixels under quadratic regularization. We chose to set the parameters $\left[\beta_{1}, \beta_{2}\right]=2 \beta_{0}$ for increased noise suppression in the $\left\{S_{1}, S_{2}\right\}$ channels. The second set of regularization parameters $\left[\delta_{0}, \delta_{1}, \delta_{2}, \delta\right]$ was determined by sweeping each parameter over a range of values and choosing the

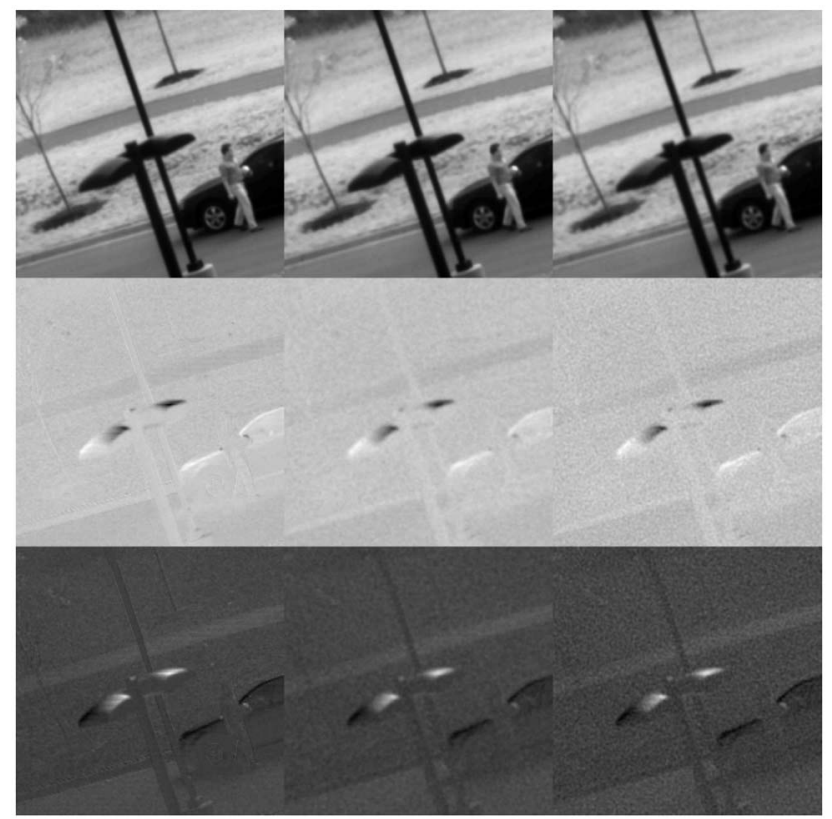

Fig. 2. Estimates of Stokes images for $\mathrm{SNR}=25 \mathrm{~dB}$. All rows read from left to right: pristine, proposed method, traditional method. First row, $S_{0}$; second row, $S_{1}$; third row, $S_{2}$. parameters that yielded a minimum RMS error in the estimate. The cross-channel regularization was evaluated for the Stokes estimator with channel-by-channel edgepreserving regularization. The optimal $\delta$ values were used and the parameter $\beta_{\text {cross }}$ was swept over a range to determine an optimal setting. Once the optimal values of the regularization parameters were determined for both data SNR levels, the estimator was evaluated over a 100realization noise ensemble.

\section{RESULTS}

Tables 1 and 2 compare the RMS estimation errors of four quantities over the noise ensemble: (1) the $S_{0}$ estimate, (2) the $S_{1}$ estimate, (3) the $S_{2}$ estimate, and (4) the estimate of the degree of linear polarization (DOLP). The DOLP is a useful quantity in polarimetric image analysis and is defined by DOLP $=\sqrt{S_{1}^{2}+S_{2}^{2}} / S_{0}$.

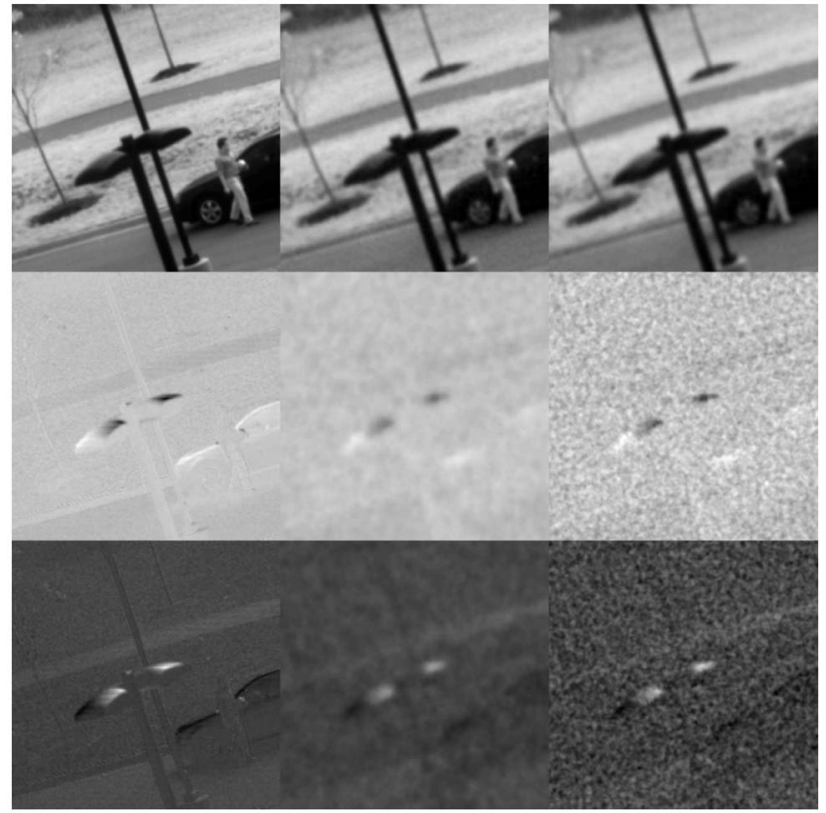

Fig. 3. Estimates of Stokes images for $\mathrm{SNR}=15 \mathrm{~dB}$. All rows read from left to right: pristine, proposed method, traditional method. First row, $S_{0}$; second row, $S_{1}$; third row, $S_{2}$. 


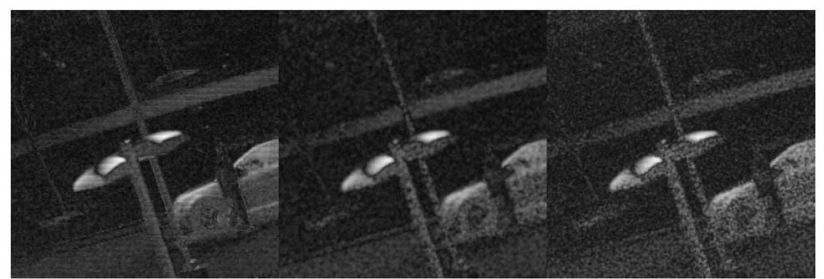

Fig. 4. Estimates of the DOLP for $\mathrm{SNR}=25 \mathrm{~dB}$; from left to right: pristine, proposed method, traditional method.

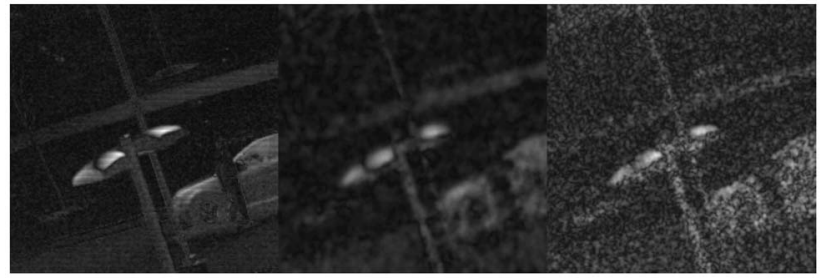

Fig. 5. Estimates of the DOLP for $\mathrm{SNR}=15 \mathrm{~dB}$; from left to right: pristine, proposed method, traditional method.

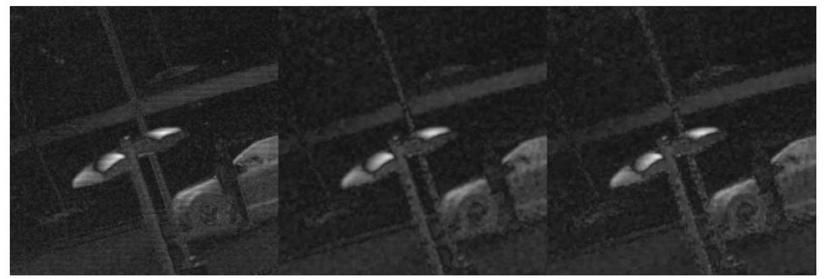

Fig. 6. Estimates of the DOLP for $\mathrm{SNR}=25 \mathrm{~dB}$; from left to right: pristine, proposed method with cross-channel regularization, traditional method with cross-channel regularization.

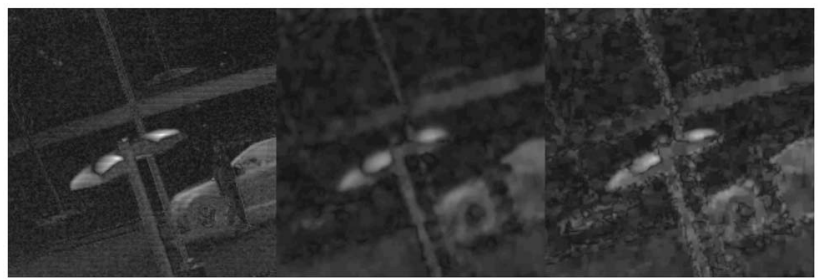

Fig. 7. Estimates of the DOLP for $\mathrm{SNR}=15 \mathrm{~dB}$; from left to right: pristine, proposed method with cross-channel regularization, traditional method with cross-channel regularization.

The proposed and traditional estimators both perform well in the estimation of $S_{0}$ at both SNR levels. However, the proposed estimator outperforms the traditional estimator, especially in the $15 \mathrm{~dB}$ SNR case, on the $S_{1}$ and $S_{2}$ images as well as the DOLP. The superior performance of the proposed estimator can be attributed to the joint nature of the estimator in which regularization is applied directly to the Stokes images. The addition of the crosschannel regularization term improves both estimators. The cross-channel term improved the traditional estimator more than the proposed estimator in RMS error because of the higher correlation between the intensity channels than between the Stokes channels. The addition of the cross-channel regularization term brought the estimators into near equivalent performance in the high SNR case. In the low SNR case the cross-channel regularization helped both estimators but the proposed estimator maintained superior performance.

Figure 1 shows the noisy and blurred data for both SNR levels; Figs. 2 and 3 show estimates of the Stokes images for SNR levels of $25 \mathrm{~dB}$ and $15 \mathrm{~dB}$, respectively; and Figs. 4 and 5 show estimates of the DOLP for SNR levels of $25 \mathrm{~dB}$ and $15 \mathrm{~dB}$, respectively. Figures 6 and 7 show estimates of the DOLP with the addition of crosschannel regularization for both the proposed and traditional estimators for SNR levels of $25 \mathrm{~dB}$ and $15 \mathrm{~dB}$, respectively.

\section{CONCLUSIONS AND FUTURE WORK}

Estimation of Stokes vectors directly provides estimates with lower overall RMS error as compared with restoring the intensity images and then transforming to Stokes space for interpretation. The addition of a cross-channel regularization term improves interpretability markedly for both the proposed estimator and the traditional estimator. In the low ( $15 \mathrm{~dB}$ ) SNR regime the proposed estimator outperforms the traditional estimator both with and without cross-channel regularization. Future work will include addressing nonidealities such as aliasing and broadband optical PSF effects. Also, estimator efficiency, convergence properties, and automatic selection of regularization parameters will be investigated.

\section{REFERENCES}

1. J. S. Tyo, D. L. Goldstein, D. B. Chenault, and J. A. Shaw, "Review of passive imaging polarimetry for remote sensing applications," Appl. Opt. 45, 5453-5469 (2006).

2. H. Barrett and K. J. Myers, Foundations of Image Science (Wiley, 2004).

3. W. G. Egan, "Polarization and surface roughness," Proc. SPIE 3426, 144-152 (1998).

4. R. A. Chipman, Polarimetry, Vol. 2 (McGraw-Hill, 1995).

5. E. Hecht, Optics, 3rd ed. (Addison-Wesley, 1998).

6. J. S. Tyo and H. Wei, "Optimizing imaging polarimeters constructed with imperfect optics," Appl. Opt. 45, 5497-5503 (2006).

7. J. Zallat and C. Heinrich, "Polarimetric data reduction: a bayesian approach," Opt. Express 15, 83-96 (2007)

8. T. F. Chan and C. Wong, "Multichannel image deconvolution by total variation regularization," Proc. SPIE 3162, 358-366 (1997).

9. L. Bar, A. Brook, and K. Nahum, "Deblurring of color images corrupted by impulsive noise," IEEE Trans. Image Process. 16, 1101-1111 (2007).

10. J. R. Valenzuela and J. A. Fessler, "Regularized estimation of Stokes images from polarimetric measurements," Proc. SPIE 6814, 681403-681403-10 (2008).

11. D. Liu and J. Nocedal, "On the limited memory BFGS method for large scale optimization," Math. Program. 45, 503-528 (1989).

12. J. Fessler and W. Rogers, "Spatial resolution properties of penalized-likelihood image reconstruction methods: Spaceinvariant tomographs," IEEE Trans. Image Process. 5, 1346-1358 (1996)

13. J. Fessler, Department of Electrical and Computer Engineering, University of Michigan, 1301 Beal Avenue, Ann Arbor, Michigan 48109-2122, USA, is preparing a book to be titled Image Reconstruction: Algorithms and Analysis.

14. P. Blomgren and T. Chan, "Color tv: Total variation methods for restoration of vector-valued images," IEEE Trans. Image Process. 7, 304-309 (1998). 